\title{
LA PERSPECTIVA DE GÉNERO EN EL COMERCIO JUSTO: UNA REFLEXIÓN ECONÓMICO-ANTROPOLÓGICA
}

\author{
María Encarnación Quesada \\ equesada77@hotmail.com \\ Doctorante de la Universidad Complutense de Madrid y \\ del Instituto Tecnológico de Monterrey, Campus Chiapas
}

\section{RESUMEN}

El Comercio Justo como estrategia de desarrollo cuenta entre sus criterios de certificación la igualdad entre hombres y mujeres. Tras un trabajo de campo realizado en Chiapas, se observa que las productoras de café obtienen más beneficios económicos que en el mercado tradicional, sin que ello suponga un empoderamiento en su entorno. Entre las fortalezas para llevar a cabo este criterio encontramos la organización de las mujeres como base para su desarrollo, la sensibilización en cuanto a la desigualdad entre los géneros, el acceso a los recursos y la identificación de las mujeres como sujetos autónomos; entre las debilidades, la ideología del sistema patriarcal, las dificultades de desplazamiento de las mujeres, el monolingüismo o la asunción naturalizada de su multiplicidad de roles: productoras, cuidadoras, madres y esposas.

Palabras clave: comercio justo, género, empoderamiento, economía.

\begin{abstract}
As a development strategy fair trade includes among its certification criteria equality of rights between men and women. After a visit to the field in Chiapas, the study observed that coffee women producers obtain certainly more financial benefits with certified product rather than with the traditional market. However, this does not imply an empowerment at the level of local communities. Several advantages to applying the equality criterion were found. These include the organization of women as a starting
\end{abstract}


point for development, the raising of awareness of gender inequality, gender training for women, access to resources and the identification of women as independent subjects. The disadvantages of this approach included those elements that upheld the ideology and the patriarchal system, the difficulties women experienced in moving from one place to another, monolingualism, domestic violence and the natural multiplicity of their roles as producers, carers, mothers and wives.

Key words: fair trade, gender, empowerment, economic.

\section{INTRODUCCIÓN}

El Comercio Justo es un mercado perteneciente a las economías alternativas o solidarias. Se trata de una comercialización de productos Sur-Norte, que se realiza con unas reglas diferentes al libre mercado en cuanto a los actores implicados, finalidades, condiciones y principios. Cuenta con un componente ideológico, posicionándose contra el neo-liberalismo con el objetivo de cambiar las injustas reglas del comercio internacional y someter al comercio a las necesidades de los pueblos y de los sectores oprimidos.

A través de un trabajo de campo realizado en cooperativas cafetaleras de Chiapas, México, certificadas con Flo Cert, ${ }^{1}$ he analizado uno de sus criterios para la certificación de los productos, la igualdad entre mujeres y hombres, arrojando luz sobre la realidad de su cumplimiento, basado prácticamente en la participación de ellas en las directivas o cargos de decisión, sin ningún cuestionamiento sobre el sistema patriarcal o incidencia en las causas de opresión de las mujeres.

El objetivo general es analizar si efectivamente el Comercio Justo lleva a cabo este criterio, cuál es su sustento teórico y si una determinada medida tiene una incidencia 
directa sobre el empoderamiento de las mujeres y, por lo tanto, una transformación real de su situación de sumisión.

Cuando me inicié en esta temática, los indicadores que encontraba hacían referencia fundamental y únicamente a la igualdad salarial, así que empecé a plantearme cuál era la base teórica que sustentaba este criterio y cuáles eran sus concepciones en cuanto al género y a la igualdad, parte del cuerpo teórico de esta investigación.

Para poder responder a estas preguntas he realizado un análisis holístico sobre la situación de la economía internacional y los mercados globales, el acceso de las mujeres a los mercados y sus condiciones de trabajo, las relaciones de poder que se establecen en la familia y en la empresa, la dicotomía público-privado, el liderazgo de las mujeres empleadas, las políticas de desarrollo, así como otros aspectos que quedan al descubierto a través del método etnográfico de investigación.

\section{ECONOMÍA Y GÉNERO}

La economía nos ha sido presentada a lo largo de la historia como algo neutro, cuando lo neutro, en un sistema patriarcal, ${ }^{2}$ es creado desde lo masculino, como eje hegemónico que vertebra todos los sistemas de la realidad.

Al referirnos a Cristina Carrasco (2003), las investigaciones en economía aparecen como representaciones abstractas del mundo llevadas a cabo por investigadores donde se omite y excluyen a las mujeres y a la actividad fundamental que estas realizan, aunque en los últimos años sí encontramos estudios relativos al trabajo doméstico, sobre la participación de las mujeres en los mercados de trabajo, o en lo relacionado con las discriminaciones laborales, y minoritariamente los que cuestionan el sesgo androcéntrico de la economía. 
En este sentido, las teorías feministas no aportan otra economía sino que ofrecen herramientas teóricas para replantear una economía más acorde con la realidad. Se trata de ponernos unas «gafas» con las cuales podamos ver el trabajo que realizan hombres y mujeres, y, más aún, que nos permitan argumentaciones que justifiquen las relaciones de poder que se establecen entre ambos.

Pero, ¿qué ocurre con el trabajo que realizan las mujeres? ¿Por qué es un trabajo que se deshistoriza, que se devalúa, se descontextualiza de la esfera laboral?

Parte de estas preguntas serán resueltas en las definiciones de género, aunque me gustaría adelantar que los géneros son definidos como opuestos, organizados jerárquicamente y construidos desde la mirada masculina.

En estos opuestos es creada la dicotomía público-privado, de manera naturalizada, relegando al hombre al ámbito público y a las mujeres al privado, base de la división sexual del trabajo y donde la economía parece estar ciega. Así, en ocasiones se presentan los deseos de las mujeres pertenecientes al ámbito privado elegidos por ellas mismas, naturalizándolos y dándolos por sentado, sin ningún tipo de crítica. Como argumenta Cristina Carrasco (2003): «La dedicación de las mujeres a la familia no está relacionada necesariamente con sus preferencias o su productividad. A menudo les viene impuesta por la renuncia de otros miembros de la familia a colaborar en el trabajo doméstico y las responsabilidades del cuidado de los niños y niñas» (2003:110).

Además, estas esferas aparecen sujetas a un orden jerárquico que sitúa al ámbito privado dependiente del público, donde estas armonizan dos estadios respectivamente: lo femenino y lo masculino, y agregan valores distintos, dado que las actividades masculinas se consideran mucho más importantes que las asignadas a las mujeres.

No se trata de que las mujeres, dentro de esos opuestos deseemos formar parte del mercado laboral asumiendo los símbolos masculinos como mejoras de nuestras 
condiciones de vida y modelo de liberalización de la opresión. Se trataría, por un lado, de que la economía visibilice el trabajo llevado a cabo por las mujeres y, por otro, de deconstruir los conceptos y teorías patriarcales para crear otros más acordes con realidades integradoras.

Por otra parte, sería reduccionista el planteamiento materialista que sitúa en el centro de las desigualdades entre los géneros la incorporación de las mujeres a los mercados laborales o el control sobre los medios de producción, porque no está teniendo en cuenta otros aspectos culturales de opresión del patriarcado, como el tipo de sexualidad impuesta, las dificultades de acceso a la participación política o a cumplir con sus propios deseos. Iris Young acuña el término «doble sistema» para referirse al hecho de que la opresión de las mujeres no se reduce solo a la economía.

Para finalizar, la división de los espacios público-privado, conlleva efectos de discriminación, pero por otra parte limita nuestro análisis, además de fragmentarlo, por lo que se hace necesario una nueva revisión que lo reconceptualice. Además de la necesidad de una ampliación del foco de la economía que va más allá de la aportación de los mercados, la monetarización de productos y servicios y lo ejecutado en el ámbito público.

\section{EL COMERCIO JUSTO}

¿QUÉ ES EL COMERCIO JUSTO?

Es una comercialización de productos Sur-Norte que están certificados con tal fin, la cual se realiza con unas reglas diferentes, en cuanto a actores implicados, finalidades, condiciones y principios. Cuenta con un fuerte componente ideológico, posicionándose contra las políticas neoliberales con el objetivo de cambiar las injustas reglas del comercio internacional y someter al comercio a las necesidades de los pueblos y de los sectores oprimidos. 
Estas reglas están marcadas por la FLO (Fairtrade Labelling Organizations), certificadora del Comercio Justo y de las cooperativas entrevistadas. Es una asociación constituida por 25 organizaciones $^{3}$ que apuestan por las iniciativas de sellos del Comercio Justo, formando las redes de productores, comerciantes y expertos externos. También tiene la función de inspección y sanción de las organizaciones certificadas, con la intención de garantizar a los países del norte el cumplimiento de tales exigencias.

Los criterios $^{4}$ se dividen entre genéricos, dirigidos a personas productoras y a comercializadoras, y por productos; entre los genéricos encontramos el alusivo a la no discriminación por razón de género promoviendo la igualdad y las recomendaciones para establecer el Plan de Desarrollo de Comercio Justo para pequeños productores, el empoderamiento, refiriéndose a las mujeres.

En la contraparte, FLO garantiza un precio mínimo en la compra de los productos certificados. En el caso del café significa que, aunque su precio caiga en la bolsa de Nueva York, sus productores y productoras siempre podrán contar con este mínimo establecido. Asimismo, un «premio social» es otra cantidad económica que se otorga para la inversión en la comunidad y para facilitar el cumplimiento de los criterios.

¿CUÁLES SON LAS PRINCIPALES CRÍTICAS?

Me centraré en dos tipos de críticas, una desarrollada a través del análisis del criterio de igualdad entre mujeres y hombres y otra focalizada en el debate emergente de dos movimientos del Comercio Justo, en cuanto a sus objetivos actuales.

En primer lugar, este criterio no aparece en toda la bibliografía o aparece de diferentes formas; por ejemplo, como referencia a la «igualdad salarial entre mujeres y hombres», o bien «promoviendo el trabajo de grupos más desfavorecidos como el de las mujeres». 
El resultado es que se trata de un criterio vago y difuso de difícil aplicación y evaluación, así que cada organización, productor o productora, investigador o investigadora, tiene que saber de qué trata la igualdad entre mujeres y hombres.

Como ejemplo, Francisco Vanderhoff Boersma (2006), asesor de la Unión de Comunidades Indígenas de la Región del Istmo (UCIRI) y cofundador del Mercado Justo de México, realiza la siguiente afirmación, refiriéndose al impacto de Mercado Diferente: ${ }^{5}$ «La organización de las mujeres dentro de la Unión, con sus propias empresas de producción, crea un caminar lento de independizarlas de los ingresos del marido y tener la capacidad de desarrollar empresitas propias, sean individuales, sean colectivas, lo cual ayuda en la economía familiar» (Setem, 2006:69).

Se trata de un ejemplo muy esclarecedor de la conformación ideológica del criterio: las mujeres no son percibidas como agentes de cambio de la comunidad, su identidad aparece ligada a la de un «marido», se plantean los ingresos económicos como mecanismo liberador de la opresión de las mujeres de forma «mágica» y no aparecen como sujetos económicos, sino como colaboradoras de la economía familiar, garantes de las necesidades básicas de supervivencia ${ }^{6}$ y de la soberanía alimentaria. ${ }^{7}$

Es mucho más usual encontrar proyectos específicos "para mujeres» en el Comercio Justo, ya sea como proyectos independientes, donde sí es más evidente la consecución del criterio de igualdad entre mujeres y hombres, o como parte de un proyecto específico dentro de una organización, y generalmente en el sector de las artesanías. Un ejemplo de proyecto independiente sería el de Creative Handicraft, ${ }^{8}$ llevado a cabo en la India para mujeres en desventaja social. No ofrece solo una posibilidad de empleo y comercialización, sino una capacitación e inclusión en grupos de trabajo de distinta índole. Como señala su director Johny Joseph: «es un hecho probado que el crecimiento económico no produce necesariamente bienestar social [...]. Sus ingresos han aumentado continuamente, aunque aún no son lo suficiente buenos teniendo en cuanta 
sus necesidades económicas. Los patrones de toma de decisiones en sus vidas están cambiando. Es importante ver cómo incluso en una sociedad tan patriarcal como la India se puede influir en el patrón de toma de decisiones introduciendo cambios en el estatus económico de las mujeres y realizando continuamente programas de capacitación» (Setem, 2006:96-97).

En cambio, es mucho más difícil observar la aplicación de igualdad entre mujeres y hombres en los proyectos que son dependientes de otros más generales, como los que nos encontramos en las cooperativas de nuestro estudio, y que veremos con más detalle.

En cuanto al debate emergente en los movimientos de Comercio Justo, volvemos a su definición cuando enuncio que conlleva un posicionamiento ideológico frente a las políticas económicas neoliberales.

Esther Vivas (2006) define dos grupos enfrentados en lo que respecta a objetivos y estrategias de trabajo, uno que denomina «tradicional y dominante», el cual se basa en los criterios expuestos anteriormente y cuenta con un discurso social y políticamente dominante; y otro que denomina "global y alternativo», que tiene una visión más integral y alianzas con otros movimientos sociales críticos con el modelo de globalización neoliberal.

Esta idea supone focalizar el Comercio Justo, no solo hacia la producción y comercialización, sino hacia la modificación del comercio internacional y de los procesos de globalización, algo que hasta el momento el Comercio Justo ha dejado intacto. Como veremos en el análisis de las entrevistas, este movimiento supone una tensión en cuanto al deseo de los productores y productoras, difícil de resolver. 


\section{LOS CONCEPTOS DE GÉNERO E IGUALDAD, APROXIMACIONES}

Para poder debatir sobre el criterio de igualdad entre mujeres y hombres se nos hace imprescindible analizar antes los conceptos de género e igualdad, para después introducirnos en la cuestión de ¿qué entiende el Comercio Justo que es el género y qué entiende que es la igualdad?

GÉNERO

En principio, el concepto hace alusión al sistema sexo-género: el sexo describe la diferencia biológica entre mujeres y hombres, y el género designa lo que en cada sociedad se atribuye a cada uno de los sexos; es decir, aquello que como construcción social se considera masculino o femenino (Eagleton, 2000).

Hagamos un breve recorrido histórico. En el siglo XVIII, el discurso médico se apropia de la definición de las diferencias sexuales y asume que hay una división natural de los cuerpos de los hombres y las mujeres como una dicotomía que ordena parte del mundo. Sin embargo, desde la Edad Media nos encontramos con una concepción del cuerpo llamado isomórfico - los cuerpos del hombre y la mujer son vistos como uno-, ya que no existía la dicotomía de la diferencia natural de los cuerpos. Según S. Juan de Huarte (1575), hombres y mujeres tenemos el mismo cuerpo, con exactos órganos sexuales, únicamente que en el caso de los hombres esos órganos se encuentran fuera del cuerpo, y en el de la mujer, en el interior del mismo.

A partir de entonces, nos encontramos un cuerpo dimórfico, ya no hablamos de uno solo, sino de que hombres y mujeres tenemos cuerpos diferentes a partir de nuestros órganos genitales. Con esta argumentación, rompemos la fijeza de la idea universal y 
absoluta de las diferencias biológicas entre mujeres y hombres, así como la dicotomía de los sexos.

A partir del siglo XIX, las referencias a las diferencias biológicas entre mujeres y hombres se encuentran determinadas en las células y las hormonas (Alsop et al., 2002). Las diferencias sexuales no son válidas para ofrecer ninguna causalidad, no explican las diferencias entre los géneros que justifican discriminaciones o desigualdades y obvian otras posibilidades sexuales; ${ }^{9}$ sin embargo, son utilizadas para validar la jerarquía social que mantiene a la mujer en una situación de subordinación respecto del hombre, al naturalizar los comportamientos que se nos muestran como inalterables o incuestionables.

Los códigos de género se refieren a expectativas de comportamiento social que existen para cada uno de los sexos en una época o lugar determinados. Atribuyen las formas y procesos que definen, limitan y transmiten el conjunto de modelos culturalmente disponibles para que las personas lleguen a una identificación en términos de mujer y hombre.

Tras esta exposición podemos concluir que el género no se refiere a la enumeración de las categorías hombre y mujer, sino que implica una reflexión más profunda enraizada en las relaciones de poder/sumisión entre hombres y mujeres. Por ello, nos referimos a la perspectiva de género cuando nos acercamos al objeto de estudio desde esta premisa, independientemente de que dicho objeto sean mujeres o no. Por lo tanto, el Comercio Justo no debe entender que la suma de las mujeres a los proyectos productivos, por ejemplo, está en aras de conseguir el criterio de igualdad entre mujeres y hombres, algo que resolveremos en el capítulo del análisis. 
IGUALDAD

Para articular el concepto de igualdad es imprescindible abordar el de diferencia; de hecho, a partir de estos términos se desarrollan dos feminismos teóricos, el de la igualdad y el de la diferencia.

El de igualdad procede de la Ilustración —al igual que el de diferencia son conceptos construidos socialmente- y surge con la intención de crear un nuevo espacio político en el que todos los miembros sean tratados por igual, con el objetivo de terminar con los privilegios del Antiguo Régimen, pero en realidad no crea un nuevo espacio social (Jiménez, 1995). El principio de igualdad tiene efectos de «universalización y exclusión» (Ibid.,1995), camuflados en una estratificación social que asigna a las mujeres un rol de inferioridad. La esfera doméstica forma parte del papel asignado a la mujer y la política moderna no hace eco de ello. Las diferencias sexuales son silenciadas y mantenidas. Este feminismo desarrollado en el siglo XVIII parte de que las mujeres nos encontramos en una situación de desigualdad que proviene de una estructura de dominación patriarcal.

El término de diferencia ha sido relacionado con la diferencia sexual y como algo inferior. En el siglo XIX este concepto deriva de la escuela postestructuralista francesa con un nuevo sentido: acentuar la diferencia sexual, pero no desde la inferioridad, y crear un nuevo orden simbólico más acorde con lo femenino.

Por ello, para situarnos ante una postura y una visión holística de los géneros debemos cuestionar los roles tradicionales sexuados, y repensar la construcción de la subjetividad femenina y la dicotomía de las esferas público y privado.

Siguiendo la propuesta postmodernista de deconstruir las categorías creadas por el patriarcado, propongo una definición de igualdad más acorde con la temática a tratar: «distribución equitativa de trabajos e ingresos entre mujeres y hombres, traducido en 
igualdad en la responsabilidad y cuidado de la vida humana y en la participación en el trabajo remunerado» (Carrasco, 2003:111), lo cual conllevaría a una nueva organización entre mujeres y hombres y distintas asignaciones de tareas, no la falsa idea de mujeres iguales a hombres.

\section{GÉNERO, DESARROLLO Y COMERCIO JUSTO}

Antes de hablar de desarrollo, me gustaría aclarar algunas notas sobre el mismo. Al referirme al desarrollo no lo hago desde una dimensión económica, sino desde una visión holística que incluye el desarrollo social y humano, por lo que me apropiaré del concepto de "posdesarrollo", de Lourdes Benería, que hace referencia a esta pluridimensionalidad. Como cita esta autora, parafraseando a Amarthya Sen: «el desarrollo económico no se traduce automáticamente en una mejora de las condiciones de vida para las mujeres, mientras que estas pueden mejorar sin que exista una vinculación clara con el desarrollo económico» (Benería, 2005:29).

El debate abierto, y que me propongo analizar en este apartado, es el modelo de desarrollo instaurado, con dos posturas contrapuestas, las mujeres en el desarrollo y el género en el desarrollo.

Después de los años setenta, cuando se inician las políticas actuales del desarrollo, se empieza a cuestionar el papel de las mujeres. No es hasta mediados de los ochenta que encontramos en los proyectos de desarrollo objetivos concretos referidos a esta temática, lo que llamamos la perspectiva MED (Mujeres en el Desarrollo), «añadir»a las mujeres a los proyectos. Estaría relacionado con la realización de programas de desarrollo de «arriba abajo», donde ellas aparecen como beneficiarias pasivas del mismo y donde no se cuenta con su experiencia, como si su aparición en la arena política no existiera o no «sirviera», otorgándole menos valor. Además, su concepción del género no estaría relacionada con la asunción del establecimiento de las relaciones jerárquicas de poder por parte del sistema patriarcal, sino asumiendo que las mujeres 
están en una situación de desventaja social y que es necesaria la ayuda, en muchas ocasiones, incluso fomentando el mismo rol sexuado, causante de nuestra opresión.

Podemos decir que el discurso de género y desarrollo GAD emergió en los noventa de las Conferencias de la ONU y realizaba una crítica al modelo anterior, que situaba a las mujeres como «una figura de la mujer colonizada, pobre y marginal que necesitaba ser conducida, educada, capacitada para el trabajo, y la toma de decisiones en su localidad, a la que se le podía controlar desde el punto de vista reproductivo y sexual a través de una serie de procesos de desarrollo diseñados para empoderar a la mujer» (Hartcourt, 2005:13).

La perspectiva GAD parte de que el verdadero empoderamiento de las mujeres a través de las políticas de desarrollo pasaría por el reconocimiento oficial de las mismas como agentes de cambio, el reconocimiento de sus experiencias, una fuerte incidencia de las demandas del movimiento de mujeres en las instituciones del desarrollo y un cambio significativo en las estructuras de poder patriarcal.

Las diferencias en el desarrollo entre «agregar mujeres» o aplicar la perspectiva de género en el desarrollo son sustancialmente diferentes en sus estrategias, metodologías, enfoques, soluciones y objetivos.

Con lo expuesto hasta el momento sobre el desarrollo, podemos decir que el Comercio Justo podría ser una estrategia de desarrollo y además podría aplicar una perspectiva (GAD). Esencialmente, en sus inicios se trataba de reemplazar la ayuda enviada a los países del Sur bajo la forma de donaciones, por un nuevo sistema comercial que permitiera a los pequeños productores y productoras mejorar sus condiciones de vida y desarrollar sus comunidades (Palma y Gomes, 2008:102). 
El resultado actual es muy distinto, ya que nos encontramos - como nos muestra el movimiento tradicional de Comercio Justo, que es el mayoritario- con un objetivo basado en la comercialización que, aunque está fuera de las reglas del mercado internacional, sigue sus mismos preceptos; y por otro lado con proyectos dirigidos a mujeres desde una perspectiva MED.

\section{COMERCIO JUSTO Y EMPODERAMIENTO DE MUJERES}

El empoderamiento sería un paso más hacia la perspectiva GAD, que sitúa a las mujeres como núcleo central en el diseño de estrategias y necesidades, y también de manera participativa. Según Srilatha Batliwala (1977), el término empoderamiento surge del movimiento de mujeres y «se manifiesta como una redistribución del poder, ya sea entre naciones, clases, razas, castas, géneros o individuos. Las metas del empoderamiento de las mujeres son desafiar la ideología patriarcal, transformar las estructuras e instituciones que refuerzan y perpetúan la discriminación de género y la desigualdad social y capacitar a las mujeres pobres para que logren acceso y control de la información y de los recursos materiales» (1977:188). Esta definición queda incompleta si no se incluye la capacidad de acción, la agencia; no es suficiente con que las mujeres tengan acceso a la información y los recursos, ya que no tiene por qué traducirse en una cuestión automática de «ejercer» el poder.

De acuerdo con Henrieta Moore (2009), el verdadero empoderamiento de las mujeres a través de las políticas de desarrollo pasaría por el reconocimiento oficial de las mismas como agentes de cambio, el reconocimiento de sus experiencias, una fuerte incidencia de las demandas del movimiento de mujeres en las instituciones del desarrollo y un cambio significativo en las estructuras de poder patriarcal. La problemática feminista sigue residiendo en los orígenes de la subordinación de la mujer y del dominio del varón en la esfera política de la vida social. 
Un mayor desarrollo del empoderamiento nos llevaría a hablar de adquisición de «poder propio», que estaría relacionado con el poder ejercido hacia adentro, en el que yo tomo conciencia y adquiero herramientas personales para confrontarlo; el «poder con», con el concepto de autonomía — cuanto soy de autónoma para llevarlo a cabo—, con medidas políticas y con el desenmascaramiento de la subjetividad femenina, y el «poder para», que estaría relacionado con la agencia y con la movilización colectiva (Paz y Pérez, 2004; Kabeer, 1998; Rowlands, 1997).

\section{PLANTEAMIENTO METODOLÓGICO}

El trabajo de campo de la investigación lo he realizado en el sector de producción de Comercio Justo del café, las organizaciones entrevistadas han sido cinco cooperativas y el contexto geográfico es Chiapas, México. (Véase Cuadro 1)

Cuadro 1. Personas entrevistadas en las distintas organizaciones e instituciones.

\begin{tabular}{|l|l|}
\hline Organización o institución & Cargos o funciones de las personas entrevistadas \\
\hline UNAM & 2 investigadores \\
\hline FIECH & Técnico \\
& Presidente \\
\hline La ComonYajNop Tic & Presidente \\
& Secretaria \\
& 2 productoras \\
\hline Acción Solidaria de la Sierra & Presidenta \\
& Tesorera \\
\hline Unión Majomut & Presidente \\
& Técnico \\
\hline San Fernando & Presidente \\
& Tesorero \\
& Encargada de comercialización exterior \\
\hline
\end{tabular}

Fuente: Elaboración propia. Trabajo de campo.

Aunque otro motivo importante es que el café representa un sector más masculinizado que, por ejemplo, el de las artesanías —llevado a cabo casi en su totalidad por mujeres-, en el que México también es muy representativo, y desde el cual hubiera 
sido muy accesible realizar una investigación sobre «las mujeres» en el Comercio Justo, pero que resulta más dificultoso para hacerlo desde la perspectiva de género; o sea, en este caso en relación con los hombres. Como argumenta Verena Stolcke (1992), tenemos que ser cautelosas con esta afirmación, ya que solo el análisis del poder y la subordinación puede que no nos lleve a la raíz de las desigualdades de género y únicamente nos mantengamos en el discurso superfluo de la equiparación de las mujeres con los hombres. Además, Soledad González (2005) señala que un punto metodológico importante es la necesidad de analizar el trabajo femenino en relación con el masculino.

Pero, ¿cómo incluir el género en el análisis desde este referente? Mi estudio no se basa solo en qué ocurre con las mujeres productoras de café o cuál ha sido el impacto del Comercio Justo en sus vidas o en su entorno, sino que, como recoge Caroline Moser (1993), la utilización del género la llevo a cabo a través del análisis de la subordinación mujeres-hombres.

En cuanto a las identidades que adopta cada una de las personas, y que manejo en las entrevistas semiestructuradas, son múltiples; por ello cuando entrevisto a un presidente también hago referencia a su papel como productor, al igual que cuando le toca a una presidenta; por eso resulta difícil realizar un línea divisoria clara sobre cuáles son los roles que he asignado a cada una de ellas y ellos. Otras identidades que también aparecen son las de cuidadora, madre, esposa o lideresa, como veremos en el análisis. 
Foto 1. Entrevista a productora de La ComonYajNop Tic

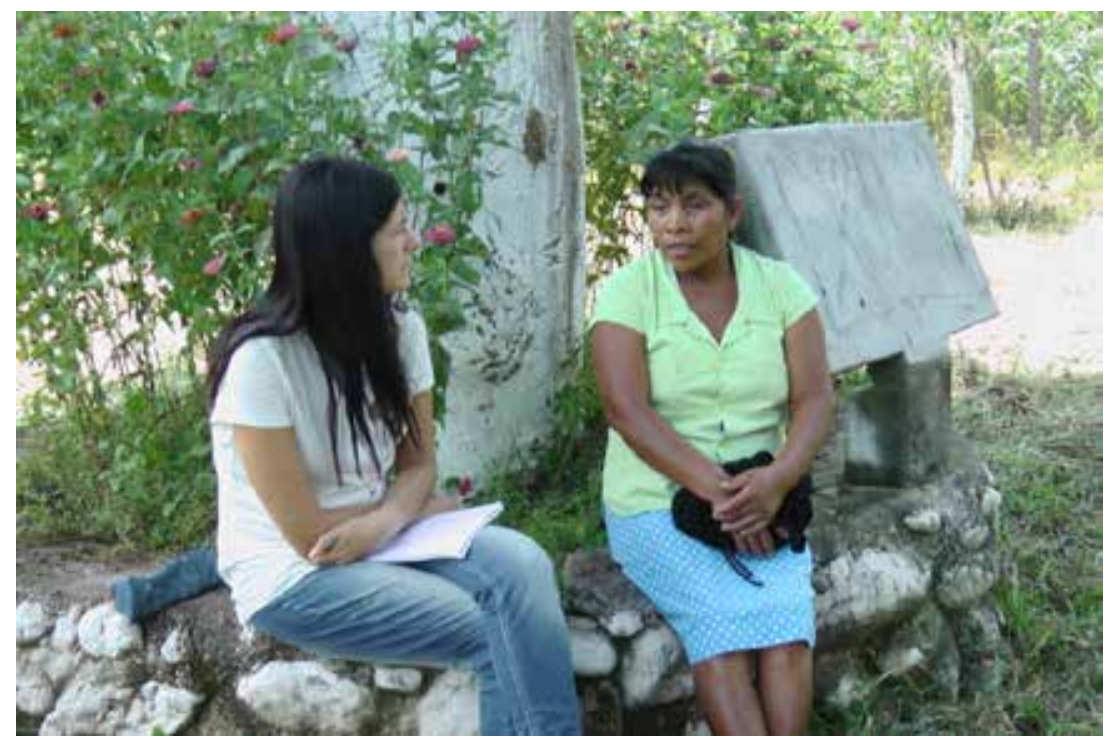

\section{ANÁLISIS DE LAS ENTREVISTAS}

Encontramos pocas diferencias fundamentales en las organizaciones entrevistadas, excepto en dos de ellas: FIECH y Acción Solidaria de la Sierra, y es que ambas tienen grupos de mujeres organizados; en cambio, en el resto tienen proyectos específicos para mujeres. También coincide, como veremos más detenidamente, que ambas organizaciones poseen una formación en género con respecto a la sensibilización sobre las desigualdades y dirigida al empoderamiento de las mujeres.

A continuación, expondré un resumen de los resultados obtenidos, sobre todo los más relevantes en cuanto a las cuestiones teóricas presentadas. 
Foto 2. Cooperativa: La ComonYajNop Tic

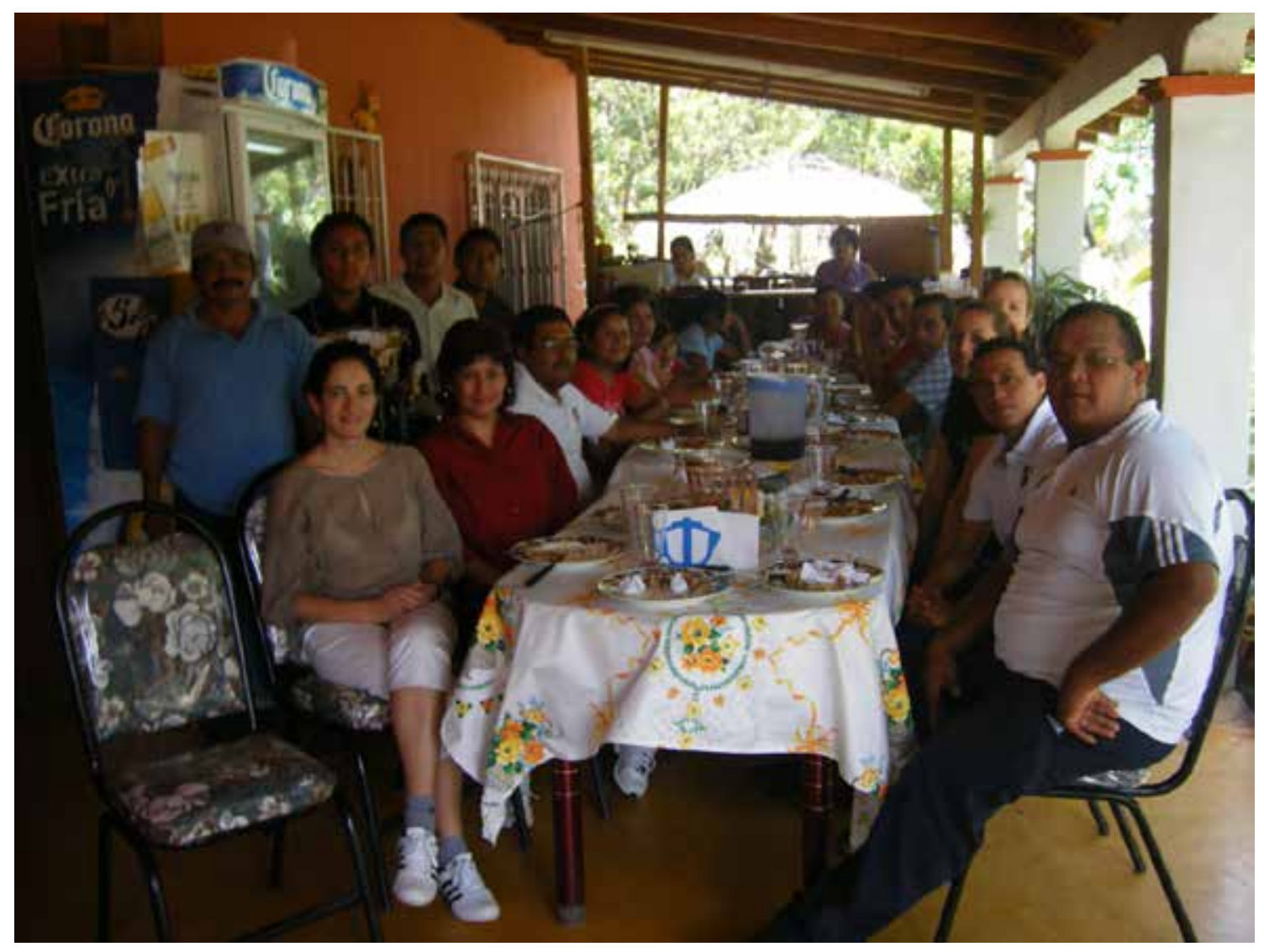

¿QUÉ ENTIENDE EL COMERCIO JUSTO POR GÉNERO?

Hay una distinción de roles que está asumida tanto por hombres como por mujeres de manera natural, la cual asigna a las últimas el trabajo doméstico y a los primeros el trabajo remunerado.

Lucía: Desde que estamos allá nos levantamos a las 4; allá tenemos que hacer el maíz, molerlo, hacer las tortillas, hacer el café, todo lo que es... de una mujer, de lo que hace una en la casa.

Baldomero: No, aquí ya, en el caso de nosotros, este... se ve más por la parte de los varones, porque aquí en la zona es muy dado que el varón salga a ganar y la mujer se quede en casa. 
Incluso, cuando se hace mención a cualidades positivas, se atienden cuestiones biologicistas, tal y como responde Vanesa cuando le pregunto cuáles son las diferencias fundamentales entre productores y productoras:

Hay pocas, y son muy buenas, y trabajan mejor, muy bien (nos reímos) y tienen las señoras mejores rentabilidades de sus tierras.

Por lo tanto, no encontramos con una definición de carácter biologicista de cada uno de los géneros, sin el cuestionamiento del constructor social que establece relaciones jerárquicas de poder entre mujeres y hombres, entre productoras y productores. En lo que el Comercio Justo no incorpora ninguna medida y lo da también por sentado.

¿QUÉ ENTIENDE EL COMERCIO JUSTO POR IGUALDAD?

Bajo el paradigma de igualdad en el que se forman los estados modernos, nos encontraríamos con la idea de que cuanto más se acerquen las mujeres al modelo de hombre (independientes, sin cargas familiares, con dedicación exclusiva al trabajo, etc.) mayor acceso a la igualdad de oportunidades tendrán; esto es validado como podremos ver en los siguientes ejemplos, aunque sería una igualdad al margen de las teorías expuestas y sin el respeto a la diversidad.

Baldomero: Eh, bueno, déjeme comentarle un caso [...] ella atiende su parcela y ella hace los trabajos de un varón, y eso es bonito porque hemos tenido ejemplo de que también la mujer puede hacer esa actividad, porque si tiene uno hijos [...] y aparte de que es soltera, porque a veces lo que ata en un matrimonio... pues el esposo ya no le permite quizá salir o por la propia obligación, porque también esto en el caso de la sociedad hay que darle tiempo, en que hay que salir y todo, y en el caso de ella tiene esa fortuna, este, de poder participar de esa manera, o sea, o también a lo mejor es por lo que mencionaba, el este...el 
prestigio, el liderazgo el que vean que la persona que es activa se carga a la gente, bueno, pongamos que la vean, que se mueve, porque parece que sí lo va a hacer.

Aquí tenemos la idea de igualdad como homogeneización; tal y como expuse, el criterio que engloba a las mujeres dentro del universo masculino:

Esteban: y de lo que decía el compañero de los proyectos que nos han dado es que va por puntos; si donde es el proyecto hay muchos indígenas pues tienes 10 puntos, si hay poco indígena un punto, y del representante también: si es el representante pues es representante de todas las mujeres, él es 1 punto y si fuera una mujer, si se hubiera puesto aquí a la licenciada, pues son 10 puntos, y todo eso no lo sabíamos nosotros.

Aunque la igualdad entre mujeres y hombres es uno de los criterios del CJ, en la práctica observamos que se trata de un criterio de «segunda», pero que suele cumplirse cuando las mujeres están organizadas, lo cual nos puede dar una clave como indicador de acción para su desarrollo, que aparece totalmente cohesionado con la participación democrática de las cooperativas.

Por otra parte, la certificadora lo evalúa teniendo en cuenta la participación numérica; por ejemplo, el número de mujeres con cargo en la dirección, un dato cuantitativo que difícilmente puede corresponder a la realidad que viven estas en esos puestos, aunque a largo plazo pueda dar sus frutos.

¿QUÉ ENTIENDE EL COMERCIO JUSTO POR PRODUCCIÓN Y QUÉ ES?

El trabajo remunerado y el no remunerado, en el caso de las productoras, aparecen interrelacionados, algo que no sucede en el caso de los productores. No se trata de un hecho aislado ya que el PNUD, en su informe de 1995, subraya la necesidad de que las 
encuestas de población activa reflejen la distribución del tiempo entre las diferentes actividades económicas que realizan los distintos miembros del hogar y las actividades que se dan de manera simultánea. Respecto a esta última afirmación tenemos los siguientes ejemplos:

Lucía: Pues ya lo que es... así, si es, por ejemplo, como una hija que tengo y me ayuda, lo que yo hago es pan, para vender con la gente, termino lo que tengo que hacer, de lavar el café, salgo corriendo para hacer pan y luego vuelvo.

Amalia: De hecho, como nos beneficiábamos en el mismo beneficio, ella (se refiere a su madre) se encargaba de la alimentación de la gente que trabaja ahí y pues yo a todo lo que era el beneficio húmedo.

Dentro de la producción también aparecen los demás miembros de la familia, como si se tratara de un trabajo colectivo. Se rompe la idea actual de la familia como unidad de consumo, para reforzar la idea de la familia como unidad de producción:

\begin{abstract}
Alberto: nunca hay dinero para el campo, está abandonado. Hoy se escucha que el campo va a ser apoyado, eso siempre se le escucha, que el campo, el campo... Pero después no es así, no llega a los productores; de hecho se les ha dicho que los funcionarios han desviado para otra parte, no sabemos para dónde han ido, todo lo que han dicho, y vuelvo a recalcar lo que aquí los compañeros, lo que es el precio justo, yo lo he comentado en las reuniones, aquí en la región no es ya rentable, lo hacemos porque lo hacemos la familia y yo, yo y la familia, pero si metiéramos jornaleros, ya no.
\end{abstract}

Nos encontramos entonces con una división total de la producción cuando está establecida la relación emocional entre productora-productor:

Baldomero: Pues del resto, en algunos casos, pues también como cuentan con el apoyo del esposo... pero ellas básicamente tienen separadas su producción. Si por decir, alguna de las señoras productoras que tiene una hectárea, 
posiblemente ayude al esposo, pero ella básicamente tiene que llevar un control; pero la mujer básicamente donde apoya es en la parte de la alimentación, de que esté pendiente de todo eso, pero sí sabe que esa parcela le está produciendo y que el café es de ella.

Vanesa: Hay señoras que tienen sus esposos que son productores, que tienen sus parcelas, y también su esposos, y entonces ellas manejan su propia producción y su acopio, y la vienen a entregar acá...

Foto 3. Cooperativa San Fernando.

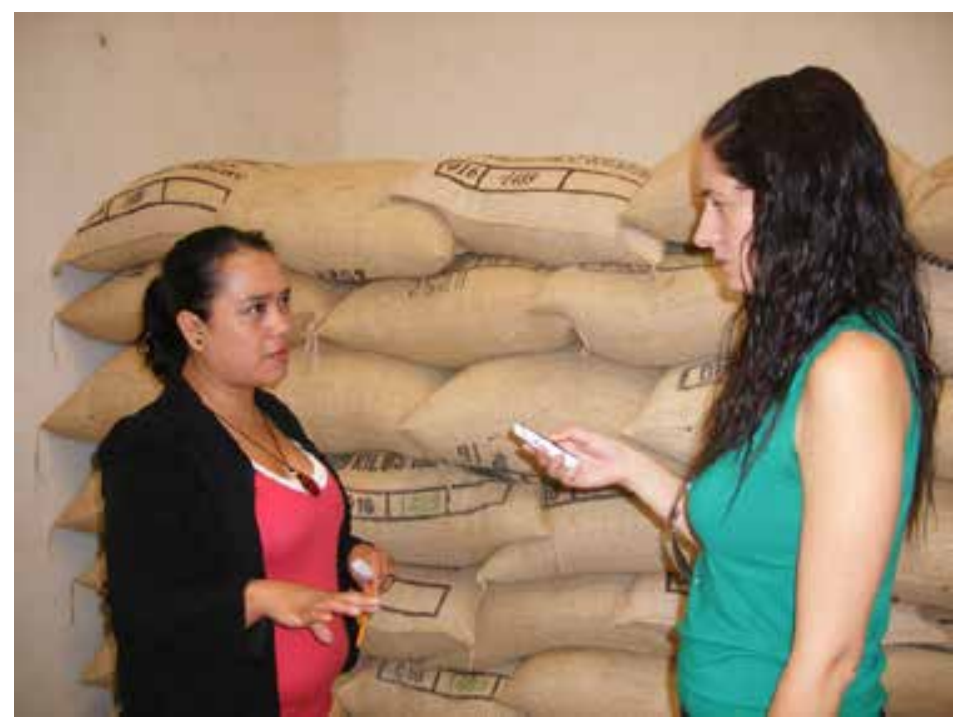

EL ACCESO A LOS RECURSOS

La igualdad en el acceso a los recursos sería un garante de que las condiciones de vida de las mujeres han mejorado. El acceso a la titularidad de la tierra es lo que ha proporcionado que las mujeres entrevistadas sean productoras y tengan ingresos propios, por lo que podría ser un elemento de empoderamiento. La mayoría de las productoras han accedido a la posesión de la tierra a través del sistema de herencia, aunque en los demás casos el acceso al recurso de la tierra lo obtuvieron a través de negociaciones en el ámbito familiar, por lo que su papel ha sido activo, frente al pasivo de transmisión de la herencia. Coincide con el hecho de que estas productoras participan 
en el consejo directivo de su organización y provienen de grupos estructurados de mujeres y con formación en género:

Mercedes: Yo me divorcié por necesidad por lo civil, y así adquirí mi terreno que era de mi marido.

Celia: Pues igual, mi esposo me proporcionó una parte de su terreno que tiene para que yo pudiera ser productora; en el momento que yo me casé, que nos casamos por todos lados, pues a mí también me tenía que tocar algo, ¿no?, entonces pues sí, de esa forma, de común acuerdo decidimos, pues tú también tienes derecho a trabajar y ahí tienes tu parte. Mi marido tampoco tiene mucho y me proporcionó una hectárea, pero sabemos que con eso podemos trabajar, y de esa forma, pues porque yo no tenía nada.

Por otra parte, observamos cómo existe un mantenimiento de la ideología patriarcal que produce una barrera para el acceso de las mujeres a los recursos, justificado con un orden jerárquico del reparto de la riqueza, en caso de herencia, de manera natural, como nos comenta Fidel:

El caso es que a veces hay un mal entendido occidentalmente, en el sentido de por qué la mujer no hereda la tierra. Si hay suficiente, pues algún trocito le han de dar a la hija o a las hijas, pero en este caso existe un regulador natural al interior de las comunidades indígenas, donde generalmente los varones son los que reciben la tierra. ¿Por qué?, Porque cuando se casa es el esposo el que va a tener la tierra; pero si el esposo no tiene tierra se la dan para que la trabaje, si no desde cuándo no hubiera más tierra acá, y entonces habría un conflicto muy fuerte por la tierra.

Garantizar la igualdad en el acceso a los recursos entre mujeres y hombres está relacionado con la promulgación de principios de justicia y derechos humanos. El problema se encuentra en su aplicabilidad, ya que el sistema patriarcal cuenta con 
argumentos que lo vulneran. El Comercio Justo y otros organismos públicos, como una ONG, deberían tenerlo en sus agendas para promover la igualdad real.

\section{LAS MUJERES Y LAS POLÍTICAS DE DESARROLLO}

La orientación que encontramos a través de las entrevistas realizadas es la de Mujeres en el Desarrollo (MED). Se han realizado proyectos productivos relacionados en su mayoría con la producción de hortalizas y aves de corral, no con el objetivo de que ellas obtengan mayor poder económico o logren ser independientes, sino para que puedan ahorrar y obtener medios de subsistencia. En este caso, las encontramos como sujeto económico, pero pasivo, lo cual dificulta, además, su entrada a una mayor participación. En una entrevista informal realizada a un asesor de una de las cooperativas, él comentaba: «desde que están los proyectos productivos, como unos ocho años, las mujeres participan menos en las directivas de las cooperativas, claro, es que están más ocupadas (se refería irónicamente a este tipo de proyectos)».

Celia: Hablábamos de los derechos de la mujer, equidad de género, salud reproductiva, lo que es la comercialización, porque también como mujeres productoras tenemos que saber cómo tenemos algunas tiendas comunitarias, también sobre administración, cuidado de los productos... todo esto también se ha dado a las mujeres.

Sin embargo, en Acción Solidaria de la Sierra encontramos otros matices en cuanto a la aplicación del género a las políticas de desarrollo. Además, es la única organización en la que encontramos capacitación en género y que promueve, a través de la formación, el liderazgo de las mujeres en las organizaciones, por lo que podríamos decir que trabajan estratégicamente desde el género en el desarrollo.

Mercedes: Estamos llevando a cabo muchísimos proyectos, hace algunos años se sacaron unos proyectos de pollos, de panadería, de granjas integrales, se han 
sacado muchos proyectos de capacitación, de los derechos de las mujeres, de equidad de género, de salud reproductiva, y muchísimos más, otros proyectos de... estamos trabajando con grupos de mujeres lo que son las artesanías. Hasta el momento tenemos algunas productoras que se dedican al bordado, al tejido en las comunidades y nosotras como organización estamos tratando de buscarles una salida, un mercado, es nuestro trabajo, ahorita, de apoyo a las mujeres porque si se están trabajando esos proyectos, también estamos trabajando con ellas en la elaboración de algunos productos domésticos para que no genere mucho egreso para su economía familiar; ahorita, en septiembre, se va a hacer un taller para enseñarle a las mujeres a hacer lo que es el cloro, la crema humectante y otros productos que sabemos que son de utilidad, para no consumir, porque nos cuesta bastante caro por esta zona y también necesitamos saber de algunas fórmulas, y entonces vamos a tratar de ver esas cosas en el taller.

Celia: Hemos conocido muchísimo, hemos conocido muchísima gente que nos ha ayudado, pero también nos han ayudado las capacitaciones; por eso siempre estamos pendientes de que se le dé capacitación a la mujer para que futuros directivos fueran mujeres, y vamos a defender esta parte, para que podamos seguir trabajando.

Esta organización forma parte de la Federación Indígena Ecológica de Chiapas, la cual nos relató cómo las mujeres participan en la elaboración de las bolsas en las que envasan el café:

Eduardo: Mire, hay un grupo de mujeres que ellas están trabajando en la elaboración de las bolsas para el envasado del café Biomasa; por ejemplo, los bolsitos que están allá (señala a una de las estanterías). (...) De la comunidad Miguel Hidalgo, entonces ellas se encargan de elaborar las bolsas, bueno, hacen muchos trabajos, ¿no?, bolsas de mano, y tienen ya el material de serigrafía, pues imprimen playeras, hacen un montón de trabajo. 
Con Acción Solidaria de la Sierra retomamos este proyecto, del que nos argumentan: «Muy buen resultado, porque ayuda y da empleo a muchas mujeres... pero ha ayudado muchísimo, como quería, el otro paso es la serigrafía, que la hicieran también las mujeres, porque hacen las bolsas y mandan hacer las serigrafías es lo que faltaría por integrar»».

Observamos que en esta ocasión tienen como objetivo que las mujeres controlen todo el proceso de producción, además de estar dirigido a la "empleabilidad" de las mismas; por lo tanto, las mujeres sí aparecen como sujeto económico.

\section{BARRERAS SEXISTAS PARA EL ACCESO DE LAS MUJERES A LA PARTICIPACIÓN}

No podemos pasar por alto la violencia de género que, sin previa estructura en la entrevista, apareció en dos ocasiones. Como nos dice Nahela Becerril: «aun cuando ha aumentado el interés y los estudios sobre la violencia de género y feminicida, la realidad no se conoce en toda su dimensión debido, por un lado a los distintos enfoques metodológicos para abordar el problema, y por otro, a la carencia institucional para el registro de información sobre casos de violencia de género contra las niñas y las mujeres en el país y en Chiapas» (2010:33). Y al ser la violencia de género la máxima expresión de la desigualdad entre los géneros, y que puede quedar encubierta con formas tradicionales-culturales de relación, el Comercio Justo debería prever este hecho.

Presento la violencia de género como barrera de participación porque está totalmente enraizada en la ciudadanía; no podemos ser ciudadanas de pleno derecho mientras sigamos apareciendo muertas en los hogares.

Otros de los elementos del sistema patriarcal que impiden la participación activa de las mujeres son la movilidad y el monolingüismo. Los desplazamientos desde los cafetales a las cooperativas o a los núcleos urbanos suelen ser largos y desde zonas de alta montaña, por lo que generalmente tienen que hacer grandes tramos a pie, con el peligro 
a la vulneración física y sexual. El transporte colectivo es prácticamente inexistente y el privado también es escaso; y si se tiene son los varones lo que poseen la licencia de conducir, por lo que los tiempos son muy importantes; si a ello le sumamos la falta de compatibilidad de la vida familiar y laboral, y la extenuante jornada (re)productiva, sus posibilidades de participación están mermadas. En toda la zona la población indígena es muy numerosa, aunque actualmente prime el mestizaje, lo cual fomenta el uso de idiolectos. El hecho de que las mujeres se encuentren más aisladas y su nivel formativo sea más bajo provoca que no hablen el castellano, fundamental para la participación para y relaciones comerciales. También de forma muy vaga, y de muy difícil reconocimiento en las entrevistas, un obstáculo de las mujeres para la participación comunitaria suele ser el rechazo por parte de sus maridos.

Eduardo: Nosotros, como organizaciones, las sedes no las tenemos en la comunidad, lo digamos, tenemos en un pueblito, donde tenemos más acceso a la comunicación... Pues entonces a una mujer se le complica más bajar de una comunidad y estar ac, y las mujeres dicen: no yo voto esto, y perfecto, ¿verdad? Y las que digan no, es que no podemos, porque se nos complica estar allí o subir y bajar, pues tampoco obligamos, ¿no?

Lucía: Pues como le digo... no tengo tiempo (para ir al centro formativo), y como muchas veces nos vamos al rancho, y como está a dos horas y media andando desde donde nos queda el carro para arriba caminando, está lejos.

Luis: Es que como no hablan muy bien el español las señoras, o sí, hay algunas que sí, pero igual no están acostumbradas a salir, por ejemplo... ¿̇Y habrá resistencia también por parte de los señores? Sí, sí, entonces, por ejemplo, es un problema.

Con esta realidad, las dificultades de participación de las productoras en las cooperativas, de manera activa, son más que claras. Teniendo presente que FloCert se centra en esta variable para valorar el alcance de la igualdad entre mujeres y hombres, 
sería recomendable que estableciera medidas para eliminar las barreras para el acceso de las mujeres a la participación.

En las siguientes tablas podemos observar qué cantidad de productores y productoras existen en las cooperativas, así como cuál es la participación de las mujeres en la directiva de la organización. Su representación es inexistente en todas, excepto en una: Acción Solidaria la Sierra es la organización que trabaja el desarrollo desde la perspectiva GAD y el empoderamiento de las mujeres. No quisiera caer en el reduccionismo que realiza el Comercio Justo a la hora de evaluar el criterio de igualdad entre mujeres y hombres, al «contar a las mujeres» sin entrar en otros criterios de carácter cualitativo o estructural, pero sí es un fiel reflejo del resultado de las barreras de participación de las mujeres.

Gráfica 1. Participación de productores y productoras socios de las cooperativas estudiadas.

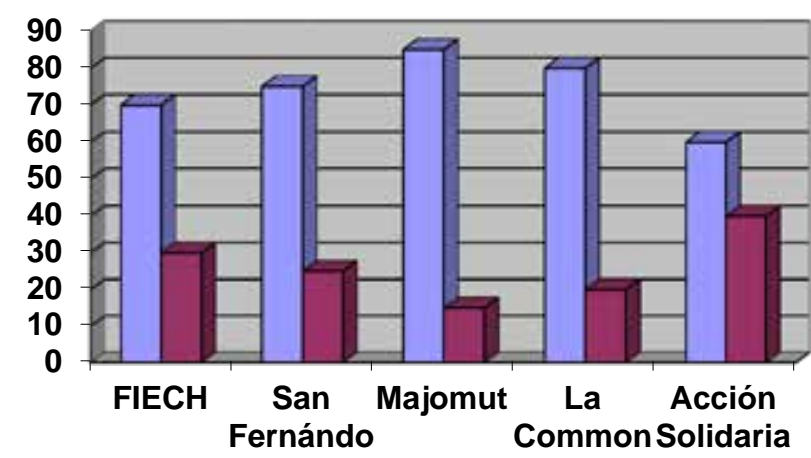

Fuente: Elaboración propia con base a datos del trabajo de campo. 
Gráfica 2. Participación de productoras socias de las directivas de las cooperativas estudiadas.

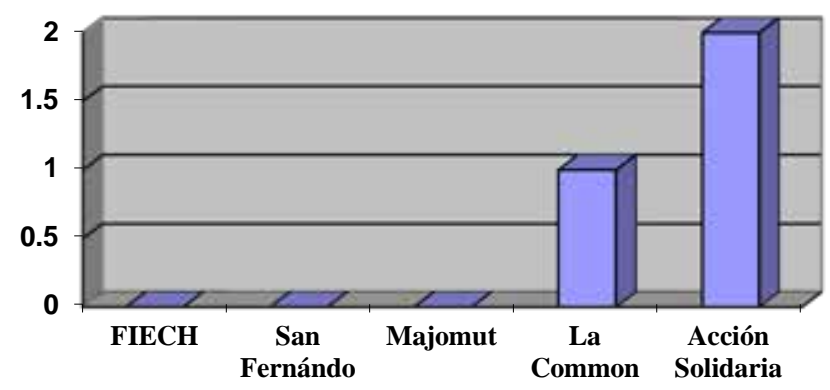

口Mujeres en la directiva

Fuente: Elaboración propia con base a datos del trabajo de campo.

Igualmente puede deberse al hecho de que las mujeres estén organizadas de manera independiente. En el caso de La Common se trata de una organización más reciente y más pequeña, por lo que su estructura es más flexible.

\section{LA ORGANIZACIÓN DE MUJERES COMO BASE PARA SU DESARROLLO}

La organización de mujeres, tanto dentro de la cooperativa como en la comunidad, parece ser un elemento clave para su desarrollo y empoderamiento. Son muy notables en las entrevistas las diferencias entre las productoras que proceden de otras organizaciones, sean de mujeres o no, pero con un matiz feminista, y las que no tienen organizaciones de mujeres en su comunidad, que son la mayoría. Los productores también aceptan que es un elemento clave para su desarrollo.

Baldomero: En las comunidades sí se organizan las mujeres para poder, inclusive si instituciones les dicen: "Les vamos a dar estos beneficios pero necesitamos que estén organizadas", pues ellas se agrupan y eso es algo que traen de beneficio a la misma familia; sí, aquí se dan en las localidades de grupos, así, de mujeres, inclusive para las cuestiones de pláticas de salud que les dan en el centro de salud.

Eduardo: Pues de igual forma yo pienso que es importante que cada organización debamos de tener en cuenta el trabajo de la mujer y que es 
importante estar organizados; antes decíamos que era difícil trabajar individualmente; pero si nosotros nos organizamos, a veces es difícil, porque nos encontramos con tantas cosas..., pero hay que buscar métodos de tal forma que la gente se vaya organizando y así facilitar el trabajo; a veces hay trabajos difíciles, como el trabajo de campo, de realizarlo una persona; pero si hacemos equipo se nos hace mas fácil, entonces invitamos a los demás grupos a que, pues, se mantenga esa unidad, y pues que no haya discriminación, pues en un grupo habemos de diferentes colores, partidos, religiones, pero es necesario mantener esa unidad sin haber discriminaciones.

\section{CONCLUSIONES}

En general, los datos que han arrojado las entrevistas han sido más que pertinentes para resolver las cuestiones planteadas inicialmente. Podemos decir que hay un conocimiento entre los productores y productoras de los procesos globales económicos y su intersección con las políticas económicas locales, aunque se trata de un tema muy complejo. Su inicio en el Comercio Justo surge por cuestiones de necesidad, tras una bajada de precios del café, por lo que se plantea como solución a los momentos de crisis y no como movimiento de incidencia política.

El planteamiento de igualdad entre los géneros, como criterio, necesita de una revisión teórica para su aplicabilidad. La certificación ha centrado su ejecución en la participación de las mujeres en los cargos directivos de las cooperativas, lo que podría ser un indicador válido para evaluar su representatividad, pero no es un fin en sí mismo. Debería centrarse en remover los obstáculos que impiden la participación de las mujeres, más que en la suma de su participación como resultado.

Los cambios proporcionados por el Comercio Justo, en sus comunidades, son escasos, aunque pudieran ser muy valiosos. Lo que aparece oculto son los cambios que pudieran 
acontecer de manera global, lo cual puede deberse a la imperceptibilidad de los mismos o a su inexistencia.

Si volvemos al inicio de la investigación, donde concretaban cuáles serían los objetivos y las preguntas de análisis, podemos concluir que el criterio de igualdad entre mujeres y hombres es vagamente conocido, y que de alguna manera se intenta llevar a cabo, por parte de las organizaciones de producción, en el sentido de equiparar a hombres y mujeres, o bien con acciones dirigidas en muchas ocasiones a fomentar su rol tradicional. Esto no cuenta con unos indicadores claros para su consecución y tendría que pensar que para ello es imprescindible un trabajo previo en la comunidad y dirigido al cuestionamiento del sistema patriarcal, como eje de opresión de las mujeres, encontrando como fuerzas favorecedoras la formación en género de las productoras, la sensibilización a las cooperativas sobre la desigualdad entre mujeres y hombres, el acceso a los recursos, la creación y fortalecimiento de las organizaciones de mujeres, su participación activa en diferentes ámbitos de la vida y la identificación de las mujeres como sujetos autónomos.

En cuanto al desarrollo, la puesta en práctica de proyectos productivo, por parte de las organizaciones productoras, para «mejorar» la situación de las mujeres sigue una perspectiva MED, que no contribuye a un cambio desde la perspectiva de género; o sea, en relación a la abolición de las relaciones jerárquicas de poder.

Para finalizar, hay que decir que el Comercio Justo por sí solo, y con este único criterio que alude a la situación de desigualdad que viven las mujeres, no tiene una incidencia directa en su empoderamiento; en cambio, cuando aparecen otros elementos como la organización previa de las mujeres, la sensibilización en cuanto a la desigualdad entre los géneros, la formación en género de las mujeres, las posibilidad de acceso a los recursos y la identificación de las mujeres como sujetos autónomos, su impacto es 
mucho mayor. En este sentido estaríamos hablando de cambios de tipo estructural, por lo que se hace necesario que estos procedan de todos los estamentos de socialización.

\section{BIBLIOGRAFÍA}

Alsop, Rachel, Annette Fitzsimons y Kathlenn Lennon, 2002, Theorizing Gender, Routledge, Londres.

Antolín, Mª José y Amaia Pérez, 2004, «El empleo femenino en la maquiladora textil en Guatemala y las transformaciones en las relaciones de género», en Globalización y desigualdad de género, compilado por Paloma de Villota, Madrid, Síntesis.

Batliwala, Srilatha, 1977, «El significado de empoderamiento de las mujeres: nuevos conceptos desde la acción», en Poder y empoderamiento de las mujeres, compilado por Magdalena León, T/M Editores, Santa Fe de Bogotá.

Becerril, Nahela, 2010, «De las palabras a los hechos, panorama de los derechos de las mujeres en Chiapas», en La equidad de género en Chiapas: teoría y práctica de la transversalidad, coordinado por Marcela Laguna, Chiapas, México, Desarrollo, Género y Ciudadanía.

Benería, Lourdes, 2005, Género, desarrollo y globalización, Editorial Hacer Barcelona.

Carr, Marilyn, 2002, «El empoderamiento económico de las mujeres: clave del desarrollo», en Empoderamiento y justicia económica. Reflexiones de la experiencia en Latinoamérica y el Caribe, coordinado por Liliana de Pauli, Ed. Mujeres-UNIFEM, México D.F. 
Carrasco, Cristina, Ed., 2003, Mujeres y economía. Nuevas perspectivas para viejos y nuevos problemas, Icaria, Barcelona.

Cavarero, Adriana, 1992, «Equality and sexual difference: Amnesia in political thought», en Beyond equality and Difference. Citizenship, Feminist Politics, Female Subjetivity, editado por Gisela Bock \& Susan James, Routledge, Londres.

Eagleton, Marie, 2000, Working with feminist criticism, Blackwell Publishers, Oxford.

Elson, Diane and Ruth Pearson, 1981, «Nimble Fingers Make Cheap Workers: An Analysis of Women's Employment in Third World Export Manufacturing», Feminist Review, 7:87-109.

González Montes, Soledad, 2005, «Las Mujeres y las relaciones de género en las investigaciones sobre el México campesino e indígena», en Estudios sobre las mujeres y las relaciones de género en México: aportes desde diversas disciplinas, coordinado por Elena Urrutia, El Colegio de México, México.

Hartcourt, Wendy, 2005, «Reflexiones sobre el movimiento global por los derechos de las mujeres», traducción de artículo publicado en la revista Development. $N^{\circ} 2$.

Hernández, Rosalva Aída, 2008, «Descentrando el feminismo. Lecciones aprendidas de las luchas de las mujeres indígenas de América Latina», en Etnografías e historias de resistencia. Mujeres indígenas, procesos organizativos y nuevas identidades políticas, coordinado por Rosalva Aída Hernández, Universidad Nacional Autónoma de México, México.

Joseph, Johny, 2006, «CJ y reducción de la pobreza. La experiencia de Creative Handicrafts», en El Comercio Justo en España, Icaria, Barcelona. 
Kabeer, Naila, 1998, Realidades trastocadas. Las jerarquías de género en el pensamiento del desarrollo, UNAM, México.

MacKinnon, Catherine, 1995, Hacia una teoría feminista del Estado, Feminismos, Madrid.

Maquierira, Virginia, 2001, «Género, diferencia y desigualdad», en Feminismos. Debates teóricos contemporáneos, coordinado por Elena Beltrán y Virginia Maquieira, Alianza Editorial, Madrid.

Mies, María, 1997, «El mito de la recuperación del retraso del desarrollo», en Ecofeminismo, teoría, cultura y perspectivas, coordinado por María Mies y Vandana Shiva, Icaria, Barcelona.

Moore, Henrietta, 2009, Antropología y feminismo, Feminismos, Madrid.

Moser, Caroline, 1993, Gender planning and development. Theory, practice and training, Routledge, New York.

Muxi, Zaida, 2009, Ciudad próxima. Urbanismo sin género, disponible en http://www.diba.cat/comerc/debats/debat1/Doc1.pdf [acceso el 27 de diciembre de 2009].

Palma, Arturo y Rosemary Gomes, 2008, «Comercio Justo y Solidario en América Latina», en El Comercio Justo en España, coordinado por Catherine A. MacKinnon, Feminismos, Madrid. 
Palmer, Ingrid, 1991, Gender and Population in the Adjustement of African Economies: Planning for Change, ILO, Ginebra.

Palmieri, Joelle y Dominique Foufalle, 2006, Feminismo y economía social y solidaria, disponible en www.ciudaddelasmujeres.com [accedido el 29 de junio de 2008].

Pateman, Carole, 1996, El contrato sexual, Anthropos, Madrid.

Riu, Héctor, 2006, «El sello de CJ en España y los pequeños productores», en El Comercio Justo en España, Icaria, Barcelona.

Rowlands, Jo, 1995, «Empoderamiento y mujeres rurales en Honduras: un modelo para el desarrollo», comunicación presentada en XIX Congreso de Asociación de estudios Latinoamericanos, Washington, 28-30 de septiembre.

Sánchez, Cristina; Elena Beltrán y Silvia Álvarez, 2001, «Feminismo liberal, radical y socialista», en Feminismos. Debates teóricos contemporáneos, coordinado por Elena Beltrán y Virginia Maquieira, Alianza Editorial, Madrid.

Scott, Joan, 1990, «Gender: An Useful Category of Historical Analysis», The American Historical Review, 91 (5), 1986, traducción española: “El género: una categoría útil para el análisis histórico", en Historia y género. Las mujeres en la Europa moderna y contemporánea, editado por Amelang, J. y M. Nash, Alfons el Magnanim, Valencia.

Sogge, David y Simon Zadek, 1998, «¿Leyes” del Mercado?», en Compasión y cálculo. Un análisis crítico de la Cooperación No Gubernamental al Desarrollo, editado por David Sogge, Icaria, Madrid.

Stolcke, Verena, 1992, Racismo y sexualidad en la Cuba colonial, Alianza Editorial, Madrid. 
Tadoro, Rosalba, 2004, «Apuntes para una conferencia: el género en el debate económico y en las políticas públicas», en Globalización y desigualdad de género, editado por Paloma de Villota, Síntesis, Madrid.

Vivas, Esther, 2006, «Los quiénes y el qué, en el movimiento del Comercio Justo», en ¿A dónde va el comercio justo? Modelos y experiencias, coordinado por Xavier Montagut y Esther Vivas, Icaria, Barcelona.

Waters, Mary-Alice, 1989, Marxismo y feminismo, Fontamara, México.

\section{NOTAS}

\footnotetext{
${ }^{1}$ La organización FLO, que expide este certificado, es explicada más adelante.

${ }^{2}$ Entiendo el sistema patriarcal compartiendo la definición de Sylvia Walby: «Un sistema de estructuras sociales interrelacionadas a través de las cuales los hombres explotan a las mujeres». En Zaida Muxi. Ciudad próxima. Urbanismo sin género. En http://www.diba.cat/comerc/debats/debat1/Doc1.pdf acceso el 27 de diciembre de 2009.

${ }^{3}$ http://www.fairtrade.net/about_us.html. acceso el 18 de noviembre de 2011.

${ }^{4}$ http://www.fairtrade.net/standards.html. acceso el 18 de noviembre de 2011.

${ }^{5}$ Se refiere a prácticas diferentes de comercialización que también toman la forma de Comercio Justo u otras denominaciones dependiendo de los países o regiones, según palabras del mismo autor.

${ }^{6}$ A través de la producción de autoabastecimiento.

${ }^{7}$ Concepto de actualidad, ligado a la crisis económica mundial y a la producción campesina, por lo que de manera inevitable tiene que aparecer en una investigación de este tipo.

${ }^{8}$ Para más información: www.creativehandicrafts.org

${ }^{9}$ Consultar: Fausto-Sterling, Anne (2006).Cuerpos sexuados. La política de género y la construcción de la sexualidad. Madrid: Melusina.
}

Fecha de recepción: 6 de diciembre de 2011.

Fecha de aceptación: 19 de febrero de 2012. 